\title{
ECOLOGY
}

\section{Snake assemblages of Marajó Island, Pará state, Brazil}

\section{Gerson Moreira Rodrigues ${ }^{1 *}$, Gleomar Fabiano Maschio² \& Ana Lúcia da Costa Prudente ${ }^{1}$}

\author{
'Departamento de Zoologia, Museu Paraense Emílio Goeldi. Caixa Postal 399, 66017-970 Belém, PA, Brazil. \\ E-mails: gerson.moreira@ifpi.edu.br; prudente@museu-goeldi.br \\ ${ }^{2}$ Instituto de Ciências Biológicas, Universidade Federal do Pará. Rua Augusto Corrêa 01, Guamá, Caixa postal 479, \\ 66075-110 Belém, PA, Brazil.E-mail: gleomar@ufpa.br \\ *Corresponding Author. E-mail: gerson.moreira@ifpi.edu.br
}

\begin{abstract}
We describe the diversity, natural history and structure of snake assemblages from Marajó Island, state of Pará, Brazil, after analyzing 439 specimens deposited in herpetological collections. We tested the hypothesis that snake assemblages from forest and open areas of Marajó Island are distinct with regard to their structure, composition and functional groups. To compare the snake composition of the forest and open areas of Marajó with other comparable assemblages in Brazil, Principal Coordinate Analysis and Clustering tests were performed. A total of 61 species of snakes was recorded for Marajó, with ten species cited for the first time for the study area (Atractus natans Hoogmoed \& Prudente, 2003, A. schach (Boie, 1827), Dendrophidion dendrophis (Schlegel, 1837), Helicops hagmanni Roux, 1910, Hydrops martii (Wagler in Spix, 1824), Lygophis meridionalis (Schenkel, 1901), Erythrolamprus typhlus (Linnaeus, 1758), Philodryas argentea (Daudin, 1803), Siphlophis cervinus (Laurenti, 1768), and Thamnodynastes sp.). The composition and structure of snake assemblages between forested and open were different, with five functional groups of snakes in forest areas, and three groups in open areas, based on habit and habitat. In all, 19 species were exclusive to forest areas, 10 were exclusive to open areas and 26 species were recorded in both areas. Our results revealed greater richness for forested areas, probably due to greater habitat heterogeneity. The species composition for forested area in Marajó was similar to that found in other Amazonian assemblages, while that for open areas was more similar to the Pantanal region than other open area assemblages. The general structure of the snake assemblage of Marajó was dominated by anurophagous, terrestrial and diurnal species. Terrestrial, arboreal and semi-arboreal snakes showed a seasonal offspring production pattern, while the pattern for aquatic and semi-aquatic species was aseasonal. The structure of assemblages was influenced by both historical and ecological factors.
\end{abstract}

KEY WORDS. Amazon, assemblage, functional groups, herpetofauna.

The characteristics of snake assemblages have been documented in studies conducted in different Brazilian biomes (VITT \& Vangilder 1983, Strussmann \& Sazima 1993, Martins \& Oliveira 1999, Marques et al. 2000, SANTos-Costa et al. 2015). Localities with a predominance of forest formations tend to have relatively higher diversity when compared to those with open vegetation (Silva \& Sites 1995, Strüssmann 2000, Bernarde \& Abe 2006, Sawaya et al.2008).

In the Amazon region, evidence of high snake species diversity has come mostly from community studies (e.g., Duellman 1978, Martins \& Oliveira 1999, Bernarde \& Abe 2006, Maschio et al. 2009) and herpetofaunistic inventories (e.g., FroTA et al. 2005, Prudente \& Santos-Costa 2005, França et al. 2006, Prudente et al. 2010, SANTOS-Costa et al. 2015). It is estimated that there are about 180 species of snakes in the Brazilian Amazon, representing 45\% of the 386 species recorded for all of Brazil (BÉRnILs \& CostA 2013).
Additional information obtained in recent years from studies on snake autoecology, with an emphasis on diet and reproduction, have begun to reveal ecological patterns for these communities (e.g., SANTOS-Costa et al. 2006, Maschio et al. 2007, Prudente et al. 2007, Albarelli \& Santos-Costa 2010, Prudente et al. 2014, SouzA et al. 2014). Nevertheless, the patterns of diet, activity and reproduction of many species are still unknown, for example, for Atractus natans Hoogmoed \& Prudente, 2003, Chironius flavolineatus (Jan, 1863), Dipsas indica Laurenti, 1768, and Eunectes deschauenseei Dunn \& Conant, 1936.

Goeldi (1897) published the first information about the herpetofauna of Marajó Island in a technical report. Almost a century later, NASCIMENTO et al. (1991), using data from the literature about reptiles from Mexiana and Marajó islands and examining material deposited in the Museu Paraense Emílio Goeldi (MPEG) and in the British Natural History Museum, 
produced a list of 38 snake species. Subsequently, YUKI \& SANTOS (1996) listed 52 species of snakes for these two islands using specimens deposited in the MPEG. This set of data resulted in a list of snake species for the island but did not include details about their structure and functional groups.

Given that Marajó Island includes both densely forested and open areas, we test the hypothesis that their snake assemblages are distinct with regard to structure, composition and functional groups. Additionally, we compared the snake composition of forested versus open areas of Marajó with comparable assemblages in other regions of Brazil. We provided more detailed information on natural history, including diet, reproduction and species distribution throughout the island.

\section{MATERIAL AND METHODS}

Marajó Island, located between the Brazilian states of Amapá and Pará $\left(0^{\circ} 59^{\prime} 39^{\prime \prime}\right.$ S, $\left.49^{\circ} 44^{\prime} 25^{\prime \prime} \mathrm{W}\right)$, has an area of approximately $49,000 \mathrm{~km}^{2}$ (GouldiNG et al. 2003), being delimited to the south by the Pará River, to the north by the Atlantic Ocean, to the east by the Marajó Bay and to the west by the mouth of the Amazon River. Three distinct ecological regions can be found on the island: dense forests predominate in the western half; successional formations or alluvial fields cover almost its entire eastern half; and Cerrado (natural grasslands) (BRITO \& ILKIU-Borges 2013) predominates in small areas of the central-north and south eastern regions of the island (RADAM 1974) (Fig. 1). Our main objective was to test if there are differences between the snakes assemblages of forested and open areas of the island. To make the comparison more robust we considered both the successional formations and the Cerrado together as comprising open habitat.

There is a distinct relationship between the vegetational distribution on the island and the mean annual temperature, with an annual mean in the eastern region $\left(27.3^{\circ} \mathrm{C}\right)$ higher than in the western region $\left(25.7^{\circ} \mathrm{C}\right)$ (Lima et al. 2005). The mean annual rainfall becomes higher from the eastern region $(<2500$ $\mathrm{mm}$ to $3000 \mathrm{~mm}$ ) towards the western region $(3000 \mathrm{~mm}$ to $>$ $4000 \mathrm{~mm}$ ). In the western half of the island, according Köppen's climate classification, the climate is Tropical Humid (Af) and in the eastern half it is Tropical Monsoon (Am) (Alvares et al. 2014).

Data was obtained from the analysis of 439 specimens from different regions of Marajó Island, collected between 1958 and 2009, and deposited in the herpetological collections of the Museu Paraense Emílio Goeldi (MPEG) and the Museu de Zoologia da Universidade de São Paulo (MZUSP) (Appendix 1), in addition to records in the literature (NASCIMENTO et al. 1991, YUKI \& SANTOS 1996).

A resource-use matrix was prepared, based on substrate use and daily activity of each species, yielding a binary matrix (SAWAYA 2008) with information on presence or absence and thus providing evidence for functional groups of forested vs. open areas of Marajó. A second matrix was prepared comparing the composition of snake species from Marajó with other forested and open formations in Brazil. Data from the literature for 10 localities were used: eastern Amazonia (Floresta Nacional de Caxiuanã and adjacent areas, Pará, Brazil - SANTOS-CosTA et al. 2015); northern

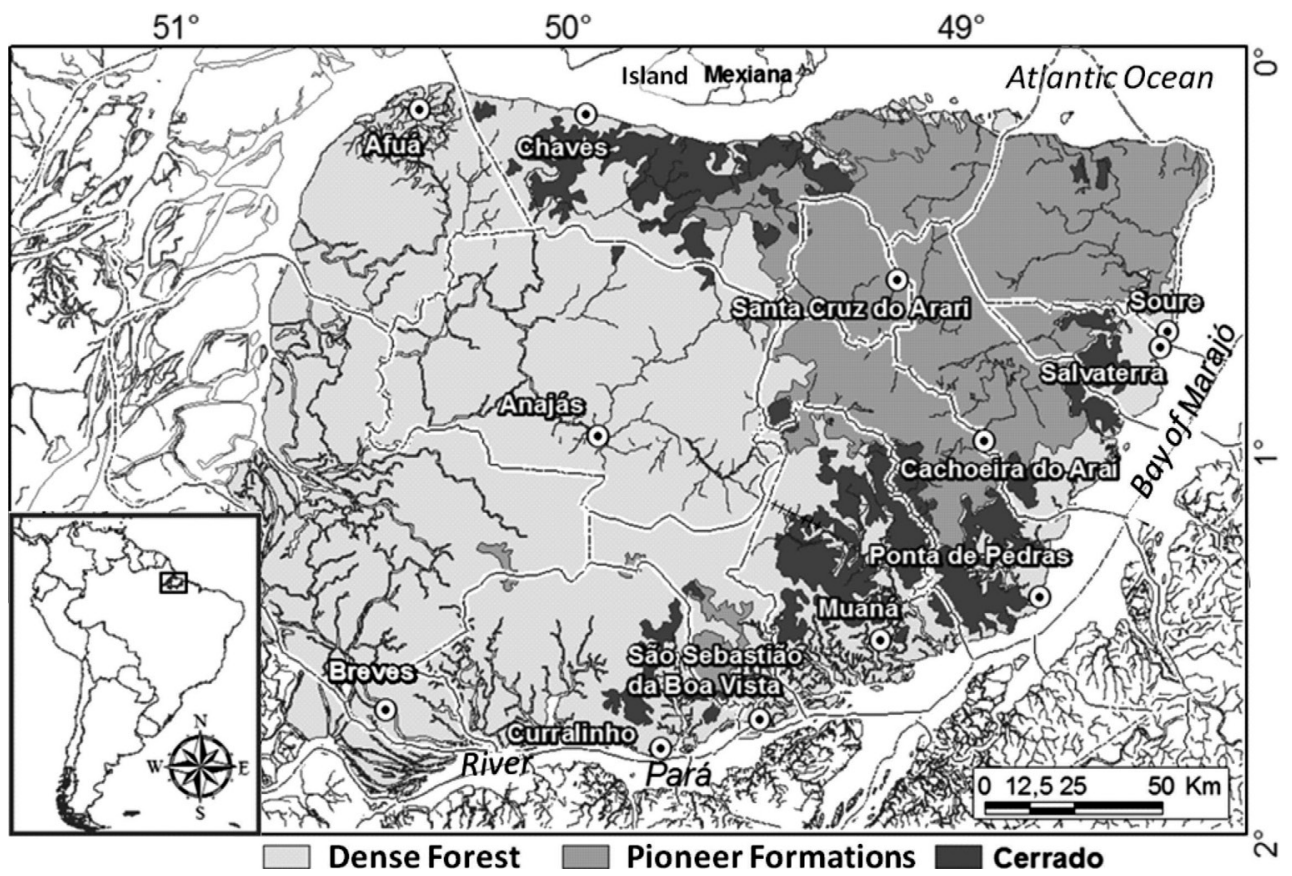

Figure 1. Map showing the location of the Marajó Island, its main plant formations and the territorial boundaries of its municipalities. Modified from Projeto RADAM (1974). 
Amazonia (French Guiana - Starace 1998); central Amazonia (Manaus, Amazonas, Brazil - MARTins \& Oliveira 1999); southeastern Amazonia (Espigão do Oeste, Rondônia, Brazil - BerNARDE \& AвE 2006); Amazonia-Cerrado transition area (Querência, Mato Grosso, Brazil - PINHeIro et al 2015); Cerrado (Itirapina, São Paulo, Brazil - SAwAYA et al. 2008; Sete Cidades, Piauí, Brazil - Rocha \& Prudente 2010); Cerrado-Caatinga transition (Castelo do Piauí, Piauí, Brazil - Rodrigues \& Prudente 2011); Caatinga (Exu, Pernambuco, Brazil - VitT \& VANGilder 1983); and Pantanal (Paconé, Mato Grosso, Brazil - StRÜssmann \& SAzImA 1993). For the resource-use matrix, we considered only the primary records of substrate used and daily activity of each species. Substrate use was considered according to the categories proposed by STRÜSSMANN (2000); aquatic or semi-aquatic snakes were included in the substrate "water", while the fossorial, cryptozoic and terrestrial snakes were included in the substrate "soil" and arboreal and semi-arboreal snakes in the substrate "vegetation". Three categories of activities were also defined: a) diurnal; b) nocturnal; c) and diurnal/nocturnal for snakes found active both during the day and night.

Cluster analysis (MANLY 1994) was performed by means of the Sorensen similarity coefficient, which gives greater weight to presence than to absence (KreBs 1989), due to the lack of information for some species. In the cluster analysis we used the Unweighted Pair-Group Method of Arithmetic Averages (UPGMA) or group mean (SNeATh \& SoKal 1973, Krebs 1989), based on the Jaccard Similarity Index (Cj). For all specimens analyzed, a longitudinal incision was made in the ventral region for observations on diet and reproduction. The presence of food content in the stomach and intestine, and prey ingestion direction was verified. The stomach contents were preserved in vials of $70 \%$ ethanol and received the same voucher number as the examined specimen. The number of prey items in each stomach was computed, and collected items were referred to specialists for identification. All prey specimens were deposited in the herpetological collection of MPEG. For reproductive characteristics, direct observations on the presence of eggs or embryos in females were carried out. Males were considered mature when the deferent ducts were opaque and convoluted, indicating the presence of sperm (SANTOS-Costa et al. 2015).

The period of offspring production (number of new offspring per individual breeder) was estimated from a circular statistical analysis (ZAR 2010), using ORIANA 4:02 (KovACH 2009). Months were converted into angles ( $30^{\circ}$ intervals) and the number of eggs, embryos and offspring observed each month was considered as their frequency in each angle (month) (Prado et al. 2005, Both et al. 2008, Prudente et al. 2014). From this method, the following values were estimated: a) mean vector $(\mu)$; b) length of the mean vector (r); c) circular standard deviation (SD) related to $\mu$; and d) Rayleigh's Uniformity Test. Rayleigh's Uniformity Test (ZAR 2010) calculates the probability of the data being evenly distributed ( $p>0.05$ ) (Null Hypothesis). A significant result of Rayleigh's Uniformity Test $(\mathrm{p}<0.05)$ indicates that data are not distributed evenly.

\section{RESULTS}

A total of 61 species of snakes were recorded for Marajó Island based on specimens deposited at MPEG $(n=423)$ and MZUSP $(n=16)$, with species distributed among six families: Boidae $(\mathrm{n}=6,9.7 \%)$, Colubridae $(\mathrm{n}=14,23 \%)$, Dipsadidae $(\mathrm{n}$ $=31,51 \%$ ) (sensu Graziottin et al. 2012), Elapidae ( $n=5,8.1 \%)$, Typhlopidae $(n=1,1.6 \%)$, and Viperidae $(n=4,6.5 \%)$ (Table 1$)$. The forested areas showed greater species diversity (45 species, including 19 exclusive species) than open areas (36 species, including 10 exclusive species).

Ten new records are cited for Marajó: Atractus natans, Atractus schach (Boie, 1827), Dendrophidion dendrophis (Schlegel, 1837), Helicops hagmanni Roux, 1910, Hydrops martii (Wagler in Spix, 1824), Lygophis meridionalis (Schenkel, 1901), Erythrolamprus typhlus (Linnaeus, 1758), Philodryas argentea (Daudin, 1803), Siphlophis cervinus (Laurenti, 1768), and Thamnodynastes sp.

Erythrolamprus poecilogyrus (Wied, 1825) was the most common species ( $\mathrm{n}=94$ specimens; $21.41 \%$ ), followed by Bothrops atrox (Linnaeus, 1758) ( $\mathrm{n}=36$ specimens; $8.2 \%)$, Erythrolamprus taeniogaster (Jan, 1863) ( $\mathrm{n}=34$ specimens; 7.74\%), Helicops leopardinus (Schlegel, 1837) ( $\mathrm{n}=25$ specimens; 5.69\%), Epicrates maurus Gray, 1849 ( $\mathrm{n}=21$ specimens; 4.78\%) and Erythrolamprus almadensis (Wagler in Spix, 1824) ( $\mathrm{n}=20$ specimens; $4.55 \%$ ). Due to the difficulty in distinguishing E. taeniogaster from Erythrolamprus cobella (Linnaeus, 1758), we considered only the presence of E. taeniogaster for Marajó, based on the distribution map presented by Fernandes et al. (2002).

It was not possible to confirm the record of Thamnodynastes strigilis (Thunberg, 1787) for Marajó, as proposed by YukI \& SANTOS (1996), because the specimen MPEG 18327 was not located. According to M.S. Hoogmoed (pers. comm.), this specimen presents a divided anal, a pre-ocular, two post-oculars and dorsals slightly keeled, insufficient characteristics to properly distinguish it from the other species of Thamnodynastes with 19 keeled dorsal rows. Seven specimens of Thamnodynastes (MPEG 20808, MPEG 20809, MPEG 20810, MPEG 20811, MPEG 21200, MPEG 21201, MPEG 21202) were not identified to species level due to the large overlap of morphological characters among species such as Thamnodynastes hypoconia (Cope, 1860), Thamnodynastes gambotensis Pérez-Santos \& Moreno, 1989, and Thamnodynastes dixoni Bailey \& Thomas, 2007 (Bailey \& Thomas 2007).

For forested areas of Marajo Island (dense forests) five functional groups were found: a group formed exclusively by aquatic species (Group A); two formed exclusively by terrestrial species (Groups B and E); and two groups formed by arboreal and semi-arboreal species (Groups C and D) (Fig. 2).

Three functional groups were found for open areas of island (successional formations and Cerrado), as follows: a group formed uniquely by aquatic snakes (Group A); a group of predominantly terrestrial snakes, but containing one arboreal species, Leptophis ahaetulla (Linnaeus, 1758), and a semi-arboreal species, Leptodeira annulata (Linnaeus, 1758) (Group B); and 
Table 1. List of species recorded, with information on diet, habitat and acitvity period in the two main phytophysiognomies of the Marajó Island, Pará, Brazil: forested areas = dense forests; open areas = pioneer formations and Cerrado. (amp) Amphisbaenians; (anu) adult anurans; (art) arthropods, except centipedes; (bir) birds; (cen) centipedes; (egb) bird eggs; (egl) lizard eggs; (fis) fishes; (liz) lizards; (mam) mammals; (mol) molluscs; (oni) onicophorous; (sal) salamanders; (sna) snakes; (tad) tadpoles; (wor) worms; (ar) arboreal; (aq) aquatic; (cr) cryptozoic; (fo) fossorial; (te) terrestrial; (d) diurnal; (n) nocturnal; (*) no information. Uppercase letters $=$ food items, habitat and activity, considered primary. Lowercase letters = food items, habitat and activity, considered occasional.

\begin{tabular}{|c|c|c|c|c|c|}
\hline & \multicolumn{2}{|l|}{ Phytophysiognomy } & \multirow{2}{*}{ Diet } & \multirow{2}{*}{$\begin{array}{c}\text { Use of } \\
\text { substrate }\end{array}$} & \multirow{2}{*}{$\begin{array}{c}\text { Daily } \\
\text { activity }\end{array}$} \\
\hline & Pioneer formations and Cerrado & Dense forest & & & \\
\hline \multicolumn{6}{|l|}{ Boidae } \\
\hline Boa constrictor Linnaeus, 1758 & 5 & 1 & BIR, LIZ, mam & $\mathrm{TE}$, ar & $\mathrm{D}, \mathrm{N}$ \\
\hline Corallus hortulanus (Linnaeus, 1758) & 1 & 3 & anu, BIR, LZ, mam & $A R$, te & $\mathrm{N}$ \\
\hline Epicrates cenchria (Linnaeus, 1758) & 0 & 1 & BIR, IIZ, mam, anu, egb & $\mathrm{TE}$, ar & $\mathrm{D}, \mathrm{N}$ \\
\hline Epicrates maurus Gray, 1849 & 21 & 0 & BIR, LIZ, mam & $\mathrm{TE}$, ar & $\mathrm{N}$ \\
\hline Eunectes deschauenseei Dunn \& Conant, 1936 & 13 & 0 & BIR, mam, FIS & $A Q$, te & $\mathrm{N}$ \\
\hline Eunectes murinus (Linnaeus, 1758) & 4 & 0 & BIR, LIZ, mam, FIS, & $A Q$, te & $N, d$ \\
\hline \multicolumn{6}{|l|}{ Colubridae } \\
\hline Chironius carinatus (Linnaeus, 1758) & 3 & 1 & ANU, bir, liz, MAM & $\mathrm{TE}, \mathrm{AR}$ & $\mathrm{D}$ \\
\hline Chironius exoletus (Linnaeus, 1758) & 1 & 1 & ANU, liz, sal & $\mathrm{TE}, \mathrm{AR}$ & $\mathrm{D}, \mathrm{n}$ \\
\hline Chironius flavolineatus (Jan, 1863) & 0 & 1 & ANU & $\mathrm{TE}, \mathrm{AR}$ & $\mathrm{D}$ \\
\hline Chironius fuscus (Linnaeus, 1758) & 0 & 5 & ANU, liz, mam, sal & $\mathrm{TE}, \mathrm{AR}$ & $\mathrm{D}$ \\
\hline Chironius multiventris Schmidt \& Walker, 1943 & 0 & 1 & ANU, LIZ, bir, mam & $T E, A R$ & $\mathrm{D}$ \\
\hline Dendrophidion dendrophis (Schlegel, 1837) & 0 & 1 & ANU, bird, liz & $\mathrm{TE}$ & $\mathrm{D}$ \\
\hline Drymarchon corais (Boie, 1827) & 1 & 0 & ANU, LIZ, SNA, MAM, egb & $\mathrm{TE}$ & $\mathrm{D}$ \\
\hline Leptophis ahaetulla (Linnaeus, 1758) & 7 & 10 & ANU, LIZ & $\mathrm{TE}, \mathrm{AR}$ & $\mathrm{D}$ \\
\hline Mastigodryas bifossatus (Raddi, 1820) & 2 & 1 & ANU, LIZ, MAM & $\mathrm{TE}$ & $\mathrm{D}$ \\
\hline Mastigodryas boddaerti (Sentzen, 1796) & 0 & 3 & ANU, LIZ, MAM & $\mathrm{TE}, \mathrm{ar}$ & $\mathrm{D}$ \\
\hline Oxybelis aeneus (Wagler, 1824) & 1 & 1 & ANU, LIZ, bir & $A R$, te & $\mathrm{D}$ \\
\hline Oxybelis fulgidus (Daudin, 1803) & 1 & 4 & BIR, LIZ & AR, te & $\mathrm{D}$ \\
\hline Spilotes pullatus (Linnaeus, 1758) & - & - & BIR, ANU, LIZ, MAM, EGB & $T E, A R$ & $\mathrm{D}$ \\
\hline Tantilla melanocephala (Linnaeus, 1758) & - & - & cen, art & $\mathrm{CR}$, te & $\mathrm{D}, \mathrm{n}$ \\
\hline \multicolumn{6}{|l|}{ Dipsadidae } \\
\hline Atractus natans Hoogmoed \& Prudente, 2003 & 0 & 4 & WOR & FO & $\mathrm{D}$ \\
\hline Atractus schach (Boie, 1827) & 0 & 1 & WOR & FO & $\mathrm{D}, \mathrm{N}$ \\
\hline Clelia clelia (Daudin, 1803) & 1 & 0 & SNA, liz, mam & TE & $\mathrm{D}, \mathrm{N}$ \\
\hline Dipsas indica Laurenti, 1768 & - & - & $\mathrm{MOL}$ & $\mathrm{AR}$ & $\mathrm{N}$ \\
\hline Drepanoides anomalus (Jan, 1863) & 1 & 0 & ANU & TE & $\mathrm{D}$ \\
\hline Erythrolamprus almadensis (Wagler in Spix, 1824) & 20 & 0 & ANU, tad, liz & $\mathrm{TE}, \mathrm{cr}$ & $\mathrm{D}, \mathrm{n}$ \\
\hline Erythrolamprus poecilogyrus (Wied, 1825) & 82 & 12 & ANU, tas, liz & $\mathrm{TE}$ & $\mathrm{D}$ \\
\hline Erythrolamprus reginae (Linnaeus, 1758) & 2 & 6 & ANU, tad, FIS & $\mathrm{TE}, \mathrm{AQ}$ & $\mathrm{D}$ \\
\hline Erythrolamprus taeniogaster (Jan, 1863) & 24 & 10 & ANU, liz & TE & $\mathrm{D}, \mathrm{n}$ \\
\hline Erythrolamprus typhlus (Linnaeus, 1758) & 1 & 1 & LIZ, EGL & $\mathrm{TE}$, ar & $N, d$ \\
\hline Helicops angulatus (Linnaeus, 1758) & 5 & 7 & FIS, TAD, anu, liz & $\mathrm{AQ}$ & $N, d$ \\
\hline Helicops hagmanni Roux, 1910 & 0 & 1 & FIS & $A Q$, te & $\mathrm{N}, \mathrm{d}$ \\
\hline Helicops leopardinus (Schlegel, 1837) & 23 & 2 & FIS, ANU & $A Q$ & $\mathrm{D}, \mathrm{N}$ \\
\hline Helicops polylepis Günther, 1861 & 1 & 3 & FIS anu & $A Q$ & $D, N$ \\
\hline Helicops trivittatus (Gray, 1849) & 7 & 1 & FIS, wor & $A Q$ & $D, N$ \\
\hline Hydrodynastes gigas (Duméril, Bibron \& Duméril, 1854) & 7 & 0 & ANU, MAM, FIS & $\mathrm{AQ}, \mathrm{TE}$ & $\mathrm{D}$ \\
\hline Hydrops martii (Wagler in Spix, 1824) & 0 & 1 & FIS & $A Q$, te & $\mathrm{D}, \mathrm{N}$ \\
\hline Imantodes cenchoa (Linnaeus, 1758) & 1 & 3 & LIZ, anu & $A R$ & $\mathrm{~N}$ \\
\hline Leptodeira annulata (Linnaeus, 1758) & 4 & 4 & ANU, tad, liz & $\mathrm{TE}, \mathrm{AR}, \mathrm{aq}$ & $\mathrm{N}, \mathrm{D}$ \\
\hline Lygophis lineatus (Linnaeus, 1758) & 15 & 1 & ANU & $\mathrm{TE}, \mathrm{AQ}$, ar & $\mathrm{D}$ \\
\hline Lygophis meridionalis (Schenkel, 1901) & 2 & 0 & ANU & $\mathrm{TE}$ & $\mathrm{D}$ \\
\hline Oxyrhopus melanogenys (Tschudi, 1845) & 1 & 0 & LIZ, MAM & $\mathrm{TE}, \mathrm{ar}$ & $\mathrm{D}, \mathrm{n}$ \\
\hline Oxyrhopus trigeminus Duméril, Bibron \& Duméril, 1854 & 0 & 0 & LIZ, MAM & $\mathrm{TE}$ & $\mathrm{D}, \mathrm{N}$ \\
\hline Philodryas argentea (Daudin, 1803) & 0 & 4 & ANU, LIZ & $A R$ & $\mathrm{D}$ \\
\hline Philodryas viridissima (Linnaeus, 1758) & 0 & 1 & BIR, ANU, LIZ, MAM & $A R$, te & $\mathrm{D}$ \\
\hline
\end{tabular}


Table 1. Continued.

\begin{tabular}{|c|c|c|c|c|c|}
\hline & \multicolumn{2}{|l|}{ Phytophysiognomy } & \multirow{2}{*}{ Diet } & \multirow{2}{*}{$\begin{array}{c}\text { Use of } \\
\text { substrate }\end{array}$} & \multirow{2}{*}{$\begin{array}{c}\text { Daily } \\
\text { activity }\end{array}$} \\
\hline & Pioneer formations and Cerrado & Dense forest & & & \\
\hline Pseudoboa coronata Schneider, 1801 & 0 & 4 & BIR, LIZ, MAM & TE & $\mathrm{D}, \mathrm{N}$ \\
\hline Psomophis joberti (Sauvage, 1884) & 1 & 5 & ANU, LIZ & $\mathrm{TE}$, ar & $\mathrm{D}$ \\
\hline Siphlophis cervinus (Laurenti, 1768) & 0 & 1 & LIZ, anu, bir, mam & VE, te & $\mathrm{N}$ \\
\hline Siphlophis compressus (Daudin, 1803) & 0 & 1 & $\mathrm{LIZ}$ & VE, te & $N, d$ \\
\hline Taeniophallus quadriocellatus Santos-Jr, Di-Bernardo \& Lema, 2008 & 0 & 2 & $\mathrm{LIZ}$ & TE & $\mathrm{D}$ \\
\hline Thamnodynastes lanei Bailey, Thomas \& Silva-Jr, 2005 & 1 & 2 & ANU & $\mathrm{TE}, \mathrm{AR}, \mathrm{aq}$ & $\mathrm{N}$ \\
\hline Thamnodynastes sp. & 9 & 4 & ANU & $\mathrm{TE}, \mathrm{AQ}$ & $N$ \\
\hline \multicolumn{6}{|l|}{ Elapidae } \\
\hline Micrurus filiformis (Günther, 1859) & 0 & 4 & AMP, SNA & FO & $\mathrm{N}$ \\
\hline Micrurus hemprichii (Jan, 1858) & 0 & 2 & AMP, ONI, SNA & $\mathrm{CR}$, FO, te & $\mathrm{D}, \mathrm{N}$ \\
\hline Micrurus ibiboboca (Merrem, 1820) & - & - & AMP, SNA & $T E, F O$ & $\mathrm{D}, \mathrm{N}$ \\
\hline Micrurus spixii Wagler in Spix, 1824 & - & - & AMP, LIZ, SNA & $\mathrm{CR}, \mathrm{FO}, \mathrm{TE}$ & $\mathrm{D}$ \\
\hline Micrurus surinamensis (Cuvier, 1817) & 0 & 1 & FIS, liz & $A Q$, te & $\mathrm{D}, \mathrm{N}$ \\
\hline \multicolumn{6}{|l|}{ Typhlopidae } \\
\hline Amerotyphlops reticulatus (Linnaeus, 1758) & 2 & 2 & ART & FO & $N$ \\
\hline \multicolumn{6}{|l|}{ Viperidae } \\
\hline Bothrops atrox (Linnaeus, 1758) & 4 & 31 & ANU, LIZ, MAM, bir, sna, cen & $\mathrm{TE}, \mathrm{ar}, \mathrm{aq}$ & $\mathrm{D}, \mathrm{N}$ \\
\hline Bothrops marajoensis Hoge, 1966 & - & - & * & * & * \\
\hline Crotalus durissus (Linnaeus, 1758) & 2 & 3 & MAM, bir, liz & $\mathrm{TE}$ & $N, d$ \\
\hline Lachesis muta (Linnaeus, 1766) & 1 & 1 & MAM & TE & $\mathrm{N}$ \\
\hline Total of species recorded & 36 & 45 & & & \\
\hline Total of specimens deposited in the MPEG and MZUSP & 439 & & & & \\
\hline
\end{tabular}

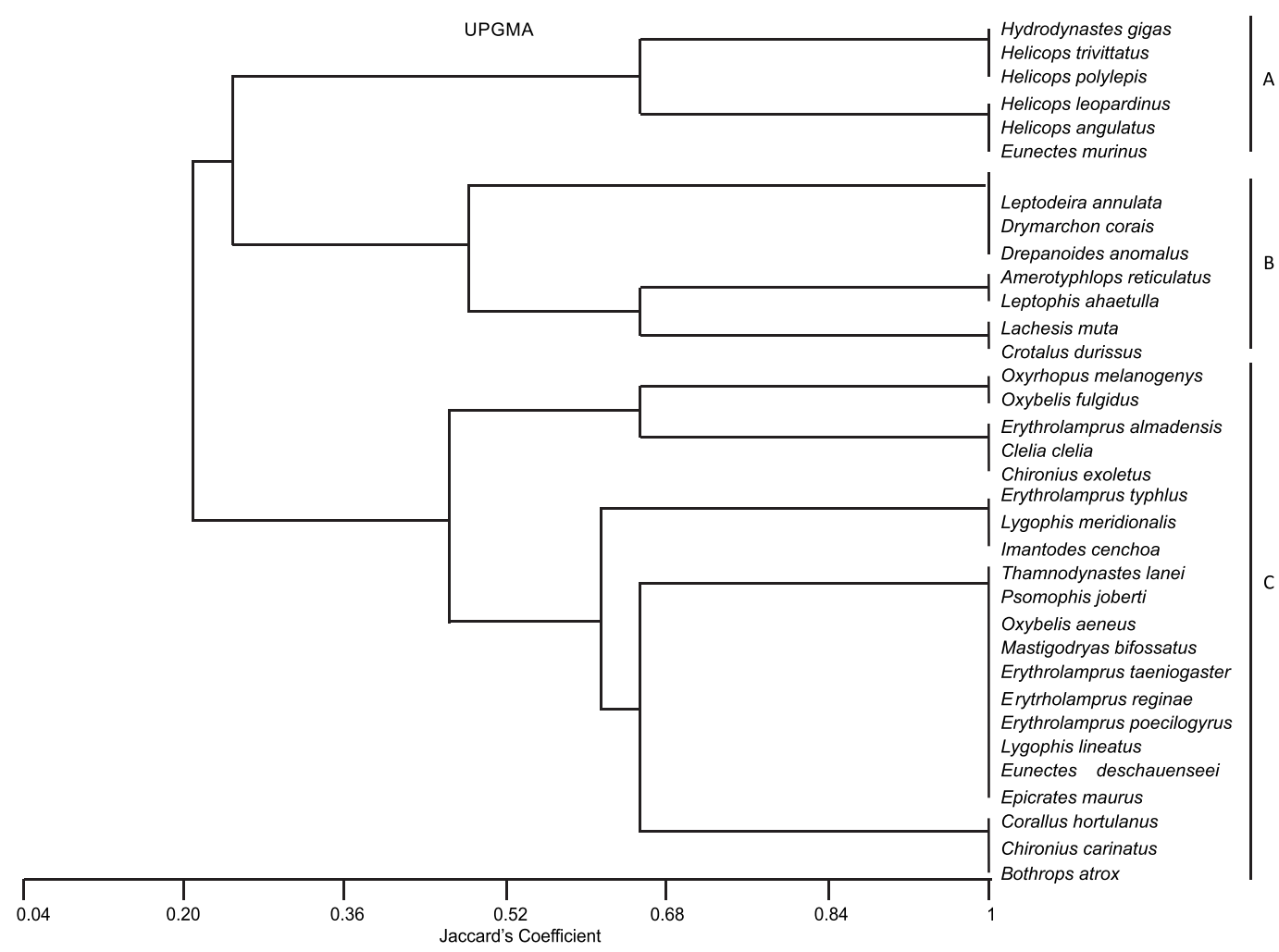

Figure 2. Grouping dendrogram of resource use based on diet and use of foraging substrate data from species recorded in open areas at Marajó Island, Pará, Brazil. 
a group of predominantly terrestrial snakes, containing four arboreal species - Oxybelis fulgidus (Daudin, 1803), Imantodes cenchoa (Linnaeus, 1758), Oxybelis aeneus (Wagler, 1824), and Corallus hortulanus (Linnaeus, 1758) - and one aquatic species, E. deschauenseei (Group C) (Fig. 3).

The aquatic group occurring in both forested and open areas is formed by nocturnal species that use the aquatic substrate for foraging a diet consisting mostly of fish. In general, its representativeness is relevant because it includes $15 \%$ of the species from Marajó - Micrurus surinamensis (Cuvier, 1817), $H$. martii, two species of Eunectes and five species of Helicops. The terrestrial groups present in forested areas are better defined than those from open areas (in which these species were grouped with arboreal ones), being formed by species that feed mainly on anurans, lizards and small mammals.

Comparing the composition of snakes from the two environments on the island with assemblages from ten other localities, we observed that the first two axes of the Principal Coordinate Analysis jointly explained $46.31 \%$ of the variation (axis 1: autovalue 1.24 and $32.69 \%$ of the variation; axis 2: autovalue 0.52 and 13.62 of the variation). The analysis ordered the assemblages into two groups: Group 1 with open area assemblages (Exú, Castelo do Piauí, and Sete Cidades); and
Group 2 formed by assemblages from predominantly forested and forest-cerrado transition areas (Poconé and open areas of Marajó; Querência; forested areas of Marajó; Espigão do Oeste; Guiana; Caxiuanã and Manaus) (Figs. 4, 5).

Of the 61 species recorded in Marajó Island, it was possible to observe the predominance of primarily or exclusively terrestrial $(36 \%, \mathrm{n}=22)$ snakes, followed by arboreal or semi-arboreal $(31 \%, \mathrm{n}=19)$, aquatic or semi-aquatic $(20 \%, \mathrm{n}$ $=12)$, fossorial $(11 \%, n=7)$ and cryptozoic $(2 \%, n=1)$ snakes, although some species used more than one substrate. Overall, 30 (49\%) species were primarily diurnal, 17 (28\%) were primarily nocturnal and 14 (23\%) could be both diurnal and nocturnal (Table 1).

A total of 369 digestive tracts from 53 species were analyzed and 119 contained some type of prey item, enabling the collection of information on the diet of 28 species. Of these, the most frequent prey item was "anuran" (56\%), followed by "mammal" (16\%), "lizard" (12\%), "fish" (8\%), "arthropod" (4\%), "earthworm" (3\%) and "snake" (1\%). Among the items that were found in adequate condition to enable the inference about ingestion direction, there was a prevalence of anteroposterior digestion ( $\mathrm{n}=36,71 \%)$ when compared to posteroanterior (n $=15,29 \%$ ) (Table 2$)$.

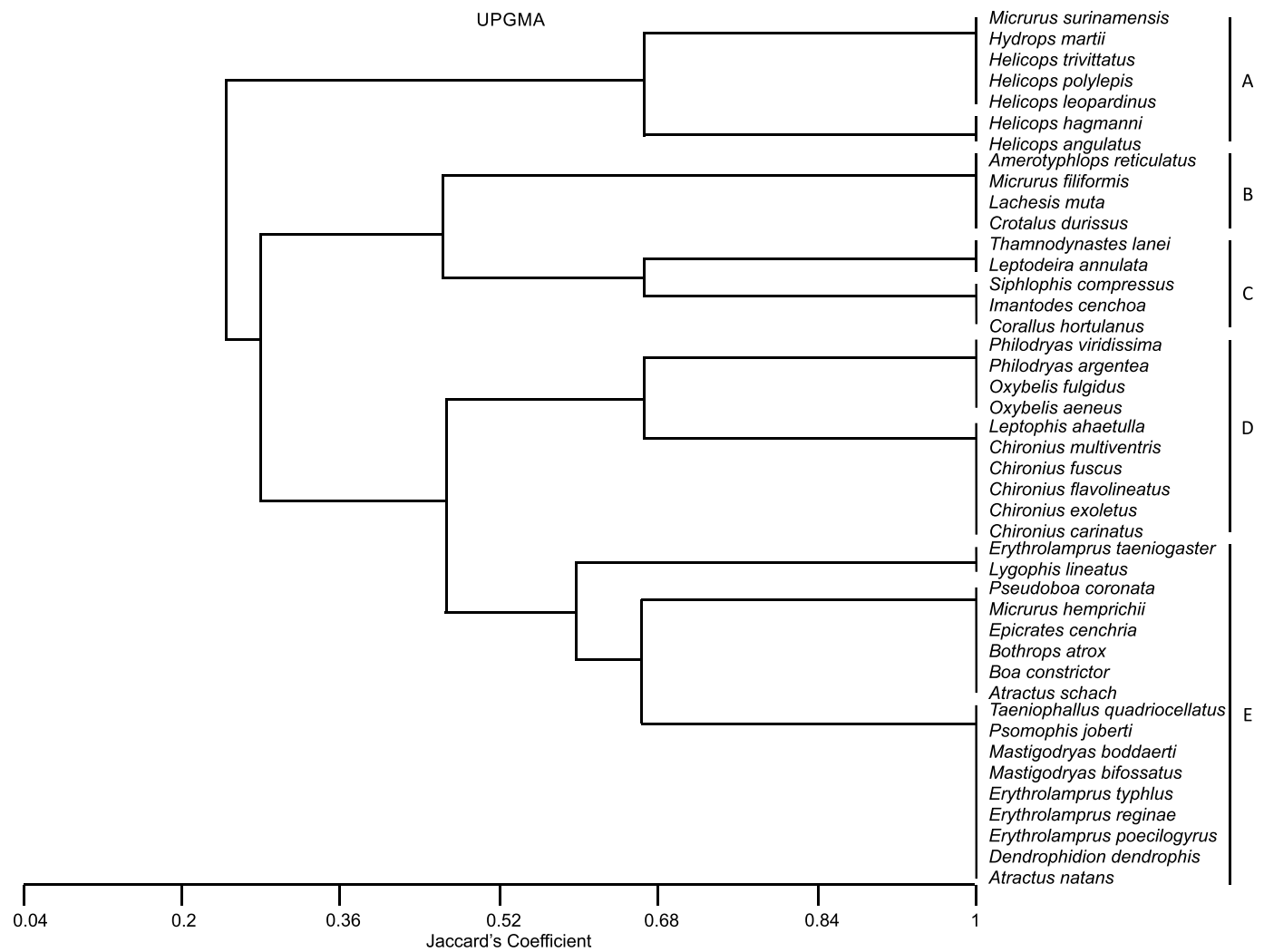

Figure 3. Grouping dendrogram of resource use based on diet and use of foraging substrate data from 61 species recorded in forested areas at Marajó Island, Pará, Brazil. 
Table 2. Food content (and respective ingestion direction) of the snakes of Marajó Island, Pará, Brazil. Legend: $n=$ number of snakes with respective content; A-P = Antero-posterior; P-A = Postero-anterior; Arabic algorism between parenthesis = number of prey in the same individual.

\begin{tabular}{|c|c|c|c|}
\hline Family/Species & $\mathrm{n}$ & Food content & Ingestion direction \\
\hline \multicolumn{4}{|l|}{ Boidae } \\
\hline Boa constrictor & 1 & Mammals (Fur) & Undetermined \\
\hline \multirow[t]{2}{*}{ Corallus hortulanus } & 1 & Mammals (Fur) & Undetermined \\
\hline & 1 & Mammal (Bat) & A-P \\
\hline \multirow[t]{2}{*}{ Epicrates maurus } & 1 & Mammal (Rodent) (2) & A-P (2) \\
\hline & 1 & Mammal (Rodent) & $A-P$ \\
\hline \multicolumn{4}{|l|}{ Colubridae } \\
\hline Chironius carinatus & 1 & Anuran (Leptoctylus petersii) (3) & P-A (2); A-P (1) \\
\hline Chironius exoletus & 1 & Anuran (Hypsiboas geographicus) & A-P \\
\hline Chironius flavolineatus & 1 & Anuran (Leptodactylus fuscus) & P-A \\
\hline Chironius fuscus & 1 & Anuran (Leptodactylus sp.) & P-A \\
\hline Chironius multiventris & 1 & Anuran (Scinax cf. $x$-signatus) & A-P \\
\hline \multirow[t]{3}{*}{ Leptophis ahaetulla } & 1 & Anuran (Hylidae) & A-P \\
\hline & 1 & Anuran (Hypsiboas sp.) & A-P \\
\hline & 1 & Arthropods & Undetermined \\
\hline Mastigodryas boddaerti & 1 & Lizard (Ameiva or Kentropyx) & Undetermined \\
\hline \multicolumn{4}{|l|}{ Dipsadidae } \\
\hline Atractus natans & 1 & Worm & Undetermined \\
\hline \multirow[t]{2}{*}{ Erythrolamprus almadensis } & 1 & Anuran (Leptodactylidae) + spider (2) & P-A (anuran) \\
\hline & 1 & Anuran & Undetermined \\
\hline \multirow[t]{8}{*}{ Erythrolamprus poecilogyrus } & 1 & Anuran (Leptodactylidae) & P-A \\
\hline & 1 & Anuran (Rhinella sp.) & $A-P$ \\
\hline & 1 & Anuran (Leptodactylus petersii) & A-P \\
\hline & 1 & Anuran (Leptodactylus macrosternum) & A-P \\
\hline & 1 & Anuran (Hypsoboas raniceps) & P-A \\
\hline & 1 & Anuran (Leptodactylus sp.) & Undetermined \\
\hline & 1 & Scale of snakes & Undetermined \\
\hline & 1 & Anuran (Tadpole) (4) & Undetermined \\
\hline Erythrolamprus reginae & 1 & Anuran (Scinax gr. ruber) & A-P \\
\hline \multirow[t]{3}{*}{ Erythrolamprus taeniogaster } & 1 & Anuran & Undetermined \\
\hline & 1 & Anuran (Tadpole) & Undetermined \\
\hline & 1 & Anuran & Undetermined \\
\hline \multirow[t]{7}{*}{ Helicops angulatus } & 1 & Anuran & P-A \\
\hline & 1 & Anuran (Rhinella margaritifera) & P-A \\
\hline & 1 & Anuran (Rhinella margaritifera) (2) & P-A (2) \\
\hline & 1 & Fish (Agenciosus sp.) & A-P \\
\hline & 1 & Fish (Rivulus sp.) & P-A \\
\hline & 1 & Fish & Undetermined \\
\hline & 1 & Fish (Astyanax cf. bimaculatus) & A-P \\
\hline \multirow[t]{4}{*}{ Helicops leopardinus } & 1 & Fish & A-P \\
\hline & 1 & Fish (Anadora sp.) & A-P \\
\hline & 1 & Anuran (Pseudis boliviana) & P-A \\
\hline & 1 & Anuran (Tadpole of Bufonidae) & Undetermined \\
\hline Helicops trivittatus & 1 & Worm & Undetermined \\
\hline Hydrodynastes gigas & 1 & Anuran (Leptodactylus macrosternum) & A-P \\
\hline \multirow[t]{3}{*}{ Lygophis lineatus } & 1 & Anuran & Undetermined \\
\hline & 1 & Anuran (Rhinella marina) (2) & A-P (2) \\
\hline & 2 & Anuran + spider & A-P (2 anuran) \\
\hline Lygophis meridionalis & 1 & Anuran (Leptodactylus fuscus or L. longirostris) & P-A \\
\hline Pseudoboa coronata & 1 & Lizards (Leposoma percarinatum) (2) + eggs (2) & A-P (2 lizards) \\
\hline Psomophis joberti & 1 & Anuran (Scinax sp.) & A-P \\
\hline Taeniophallus quadriocellatus & 1 & Lizard (Chatogekko amazonicus) & A-P \\
\hline Thamnodynastes lanei & 1 & Anuran (Hylidae) & P-A \\
\hline \multirow[t]{2}{*}{ Thamnodynastes sp. } & 1 & Insects & Undetermined \\
\hline & 1 & Anuran (Leptodactylus sp.) & A-P \\
\hline \multicolumn{4}{|l|}{ Viperidae } \\
\hline Bothrops atrox & 1 & Snakes (Bothrops atrox) Kentropyx sp. & Snakes: A-P; lizards: Undetermined \\
\hline & 1 & Lizard (Tretioscincus agilis) & A-P \\
\hline & 1 & Mammal (Fur) + Anuran & Undetermined \\
\hline & 1 & Lizard (Gymnophthalmidae, Tretioscincus sp.) +3 tooths of snakes & Undetermined \\
\hline & 1 & Lizard (Anolis sp.) +1 tooth of snakes & $A-P$ \\
\hline & 2 & Mammal (Rodent) & A-P (2) \\
\hline & 1 & Lizard (Thecadactylus rapicauda) & A-P \\
\hline & 1 & Mammal (Rodent) (3) & 3 A-P \\
\hline Crotalus durissus & 1 & Mammal (Fur) + tooths of snakes & Undetermined \\
\hline
\end{tabular}




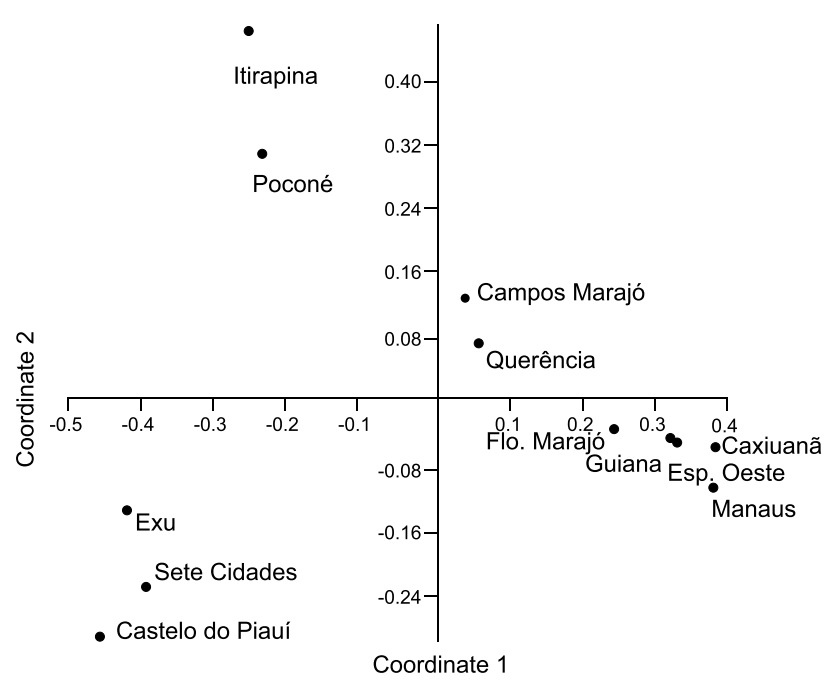

Figure 4. Ordination diagram of the Principal Coordinate Analysis resulting from the composition of snake species (presence or absence). Data for Ilha de Marajó was entered in two columns: forested area and open area. Axis 1: autovalue 1.24 and $32.69 \%$ of variance; Axis 2: autovalue 0.52 and $13.62 \%$ de variance.

Eggs, embryos and offspring of terrestrial, arboreal and semi-arboreal species were recorded mainly in the rainy season, indicating a seasonal pattern for offspring production. Eggs, embryos and offspring of aquatic and semi-aquatic species were recorded in practically all months of the year, indicating an aseasonal pattern for offspring production (Figs. 6-7, Table 3).

Table 3. Results of the circular statistical analysis testing for seasonality in the snakes offspring production at Marajó Island, Pará, Brazil.

\begin{tabular}{lcc}
\hline \multicolumn{1}{c}{ Variables } & $\begin{array}{c}\text { Eggs, embryos and } \\
\text { offspring (terrestrial, } \\
\text { arboreal and semi- } \\
\text { arboreal species) }\end{array}$ & $\begin{array}{c}\text { Eggs, embryos } \\
\text { and offspring } \\
\text { (aquatic and semi- } \\
\text { aquatic species) }\end{array}$ \\
\hline Observations $(\mathrm{n})$ & 31 & 29 \\
Mean vector $(\mu)$ & $340.86^{\circ}$ & $102.065^{\circ}$ \\
Length of mean vector $(r)$ & 0.678 & 0.158 \\
Circular Standard Deviation $(\mathrm{SD})$ & $50.518^{\circ}$ & $110.051^{\circ}$ \\
Rayleigh Test of Uniformity $(\mathrm{P})$ & $<0.0005$ & 0.488 \\
\hline
\end{tabular}

\section{DISCUSSION}

The large number of species recorded for Marajó Island reflects the high richness of snakes in the Amazon region, as demonstrated in other studies (e.g., Duellman 1978, Dixon \& Soini 1986, Martins \& Oliveira 1999, Maschio et al. 2009). In this study, the presence of ten species that had not been previously recorded for the island indicates that new records can be added even in areas that have been well inventoried (NASCIMENTO et al. 1991, YUKI \& SANTOS 1996).

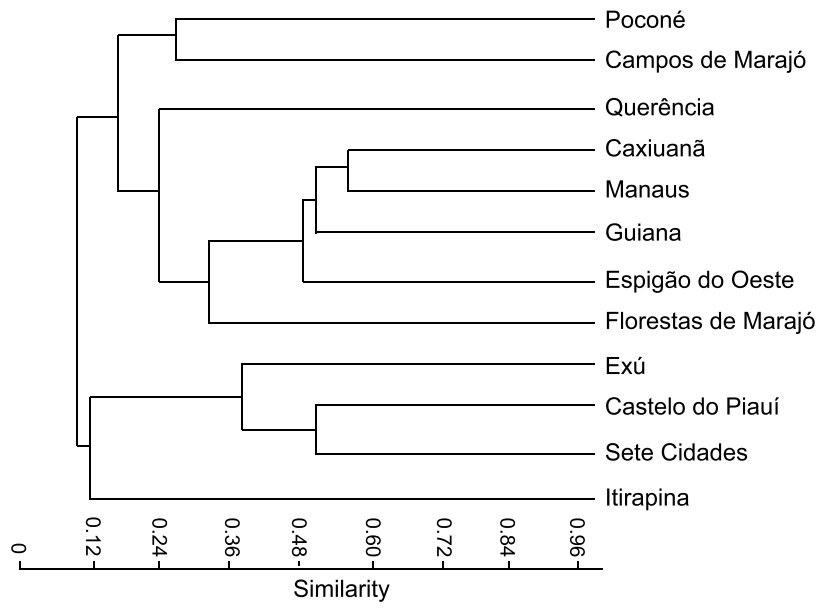

Figure 5. Grouping dendrogram resulting from the composition of snake species (presence or absence), analyzed by separating the two physionomic units (forested area and open area) in the Marajó Island, Pará, Brazil.

The higher richness of snakes observed in forested areas of Marajó appears related to the greater environmental heterogeneity. Forests have greater structural diversity of habitats, enabling snakes to occupy vertical substrates while also finding more areas of shelter and higher richness of potential prey (PIANKA 1966, ViTT 1987).

The similarity between the assemblage from forested area of Marajó and other Amazonian assemblages is the result of the high number of common species between the areas (53 species). Specifically, the high similarity of Marajó with the Caxiuanã region is probably related to the source of colonization (see MACARTHUR \& Wilson 1967), considering its recent formation and its geographical proximity to the mainland. The environmental structure of open areas of Marajó provides favorable conditions for the presence of species adapted to similar environments in other regions, such as the Caatinga of the northeast of Brazil and the Cerrado of central and northeastern Brazil.

Morphological and behavioral characteristics, as well as substrate use, foraging activity and type of prey often share a common evolutionary origin (CAdle \& Greene 1993, Alencar et al. 2013), and are fundamental in structuring snake communities (FrançA et al. 2008). Thus, snakes from the same phylogenetic lineage tend to group in dendrograms of resource use (see MARtins \& Oliveira 1999, Bernarde \& Abe 2006, Palmuti et al. 2009). This condition can be observed in Group A for the open areas of island, formed by aquatic species of Hydropsini sensu GrazzIotin et al. 2012 (Helicops and Hydrops), Hydrodynastini sensu Grazziotin et al. 2012 (Hydrodynastes gigas (Duméril, Bibron \& Duméril, 1854)) and Boinae (Eunectes murinus (Linnaeus, 1758)). Similarly, Group A for forested areas is formed by aquatic species of two phylogenetic lineages, Hydropsini (with species of Helicops and Hydrops) and Elapinae (M. surinamensis). On the 


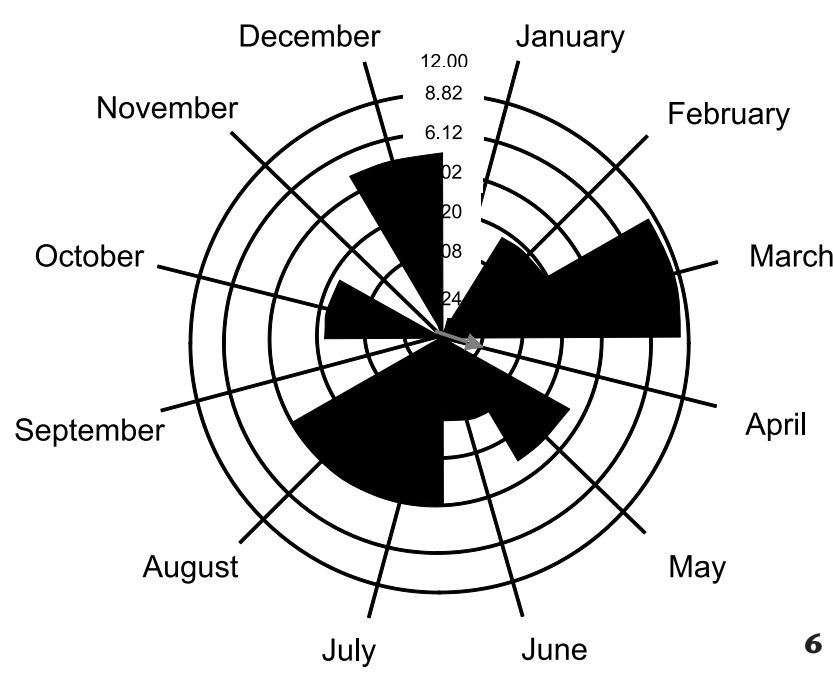

Figures 6-7. Seasonal variation in frequency of eggs, embryos and offspring of aquatic and semi-aquatic species (6) and terrestrial, arboreal and semi-arboreal species (7) in the Marajó Island, Pará, Brazil.

other hand, in grouping analysis, it was possible to observe phylogenetically distinct species that converged on the same resource, as observed, for example, in Group C for open areas formed by four subfamilies (Boinae, Colubrinae, Crotalinae and Xenodontinae) that forage both in the soil and on vegetation, preying primarily on anurans and lizards. The overlap in the use of resources by snakes from the same community indicates that factors such as prey abundance and predation may aid in avoiding competition between syntopic species (BERNARDE \& Аве 2006). Thus, the fact that there are groups formed by both phylogenetically close species and distant species may indicate that the community is being structured by both historical and ecological factors.

The record of anurans in the diet of $54 \%$ of the species from Marajó Island does not correspond to the pattern observed for central and eastern Amazonia, where a greater number of saurophagous snakes were observed, $42 \%$ and $58 \%$, respectively (Martins \& Oliveira 1999, Bernarde \& Abe 2006). The high frequency of amphibians in the diet was a result of the high frequency of species that feed primarily and exclusively on anurans, such as Chironius, Erythrolamprus and Thamnodynastes (CADLE \& GREENE 1993, Dixon et al. 1993).

The proportion of aquatic snakes on Marajó is relatively high (20\%), and is probably associated to the extensive and prolonged flood periods that occur on the island, mainly from January to June (Goulding et al. 2003). The same pattern of aquatic species (15\% of the assemblage) was observed in the Pantanal, a region that also remains flooded for long periods of the year (February to June) (Strussmann \& SAzima 1993).

In aquatic environments, seasonal variation in the availability of food resources is lower when compared to terrestrial environments (JuNK 1997). With the rise in water level during the rainy season, there is a natural expansion in the physical space and, consequently, an increase in the availability of habitats for fish prey, which reduce in density due to dispersion. However, with the lowering of the water level during the dry season, the opposite occurs, that is, the physical space decreases as does the habitat availability for prey, increasing their density (see OKADA et al. 2003, Luz-Agostinho et al. 2009). This variation in water level probably exerts some influence on aquatic species such as E. murinus, $H$. leopardinus and $H$. gigas that use fish as their main food resource (Starace 1998, López \& Giraudo 2003, Ávila et al. 2006), or on species that forage in aquatic environments such as E. taeniogaster and E. poecilogyrus, which consume tadpoles and adult anurans.

The relative ease of finding food throughout the year in aquatic environments explains the noted aseasonal offspring production for aquatic species of Marajó. The opposite situation is observed with terrestrial, arboreal and semi-arboreal species, where offspring production occurs almost exclusively in the rainy season, when the availability of resources in these substrates increases (MarTins \& OliveIra 1999).

Marajó Island corresponds to a relatively small portion of the Amazon biome, and yet its unique formation process has resulted in distinctive phytophysiological characteristics, producing environmental specialization and hence a diverse ophidofauna. The results presented here form the basis for future studies of Amazon biodiversity in a region under severe processes of fragmentation and anthropic change.

\section{ACKNOWLEDGEMENTS}

We thank Marinus S. Hoogmoed and Teresa C.S. de Ávila-Pires for identifying the amphibian and lizard content of the digestive tract, and Wolmar Wosiacki and Luiz Peixoto for identifying the fish content. We also thank Marinus S. Hoogmoed 
for lending his personal notes of the specimens from Marajó, Reginaldo Rocha for the island information and Marcelo Sturaro for helping to draw the map. We thank Ângelo Dourado, Alessandro Menks, Alexandre Cordeiro, and Luciana Oliveira for helping in the analysis of the stomach content and reproductive characteristics. We thank Anne Baldisseri and Glenn Harvey Shepard for the revision of the English. We thank Programa de Pós-Graduação em Zoologia UFPA/MPEG where GMR obtained the M.Sc. degree. We thank Conselho Nacional de Desenvolvimento Científico e Tecnológico (CNPq) for the student scholarship (process 131002/2010-4), productivity scholarship (Process 308950/2011-9 and 305475/2014-2) and financial help for the project CNPq PROTAX (56.2171/2010-0 and 44.0413/2015-0).

\section{LITERATURE CITED}

Albarelli LPP, SANTos-Costa MC (2010) Feeding ecology of Liophis reginae semilineatus (Serpentes: Colubridae: Xenodontinae) in Eastern Amazon, Brazil. Zoologia 27(1): 87-91. doi: 10.1590/ S1984-46702010000100013

Alencar LRV, Gaiarsa MP, Martins M (2013) The evolution of diet and microhabitat use in pseudoboine snakes. South American Journal of Herpetology 8(1): 60-66. doi: 10.2994/ SAJH-D-13-00005.1

Ávila RW, Ferreira VL, Arruda JAO (2006) Natural History of the South American Water Snake Helicops leopardinus. (Colubridae: Hydropsini) in the Pantanal, Central Brazil. Journal of Herpetology 40(2): 274-279. doi: 10.1670/113-05N.1

Alvares CA, Stape JL, Sentelhas PC, Gonçalves JlM, Sparovek G (2014) Köppen's climate classification map for Brazil. Meteorologische Zeitschrift 22(6): 711-728.

BAILey JR, Thomas RA (2007) A revision of the South American snake genus Thamnodynastes Wagler, 1830 (Serpentes: Colubridae, Tachymenini). II. Three new species from northern South America, with further descriptions of Thamnodynastes gambotensis Pérez-Santos and Moreno and Thamnodynastes ramonriveroi Manzanilla and Sánchez. Memoria de la Fundación La Salle de Ciencias Naturales 166: 7-27.

Bernarde PS, Abe AS (2006) A snake community at Espigão do Oeste, Rondônia, southwestern Amazon, Brazil. South American Journal of Herpetology 1(2): 102-113.

BérniLs RS, COSTA HC (2013) Brazilian reptiles. List of species. Sociedade Brasileira de Herpetologia. Available online at http://www.sbherpetologia.org.br [Accessed: 15/11/2013]

Both C, Kaefer IL, SANTos TG, Cechin STZ (2008) An austral anuran assemblage in the neotropics: seasonal occurrence correlated with photoperiod. Journal of Natural History 42(3-4): 205222. doi: $10.1080 / 00222930701847923$

Brito ES, ILkIU-Borges AL (2013) Bryoflora of the municipalities of Soure and Cachoeira do Arari, on Marajó Island, in the state of Pará, Brazil. Acta Botanica Brasilica 27(1): 124-141.

Cadle JE, Greene HW (1993) Phylogenetic patterns, biogeography, and ecological structure of neotropical snake assemblages, $\mathrm{p}$.
281-293. In: Ricklefs RE, SCHLUTER D (Eds.). Species diversity in ecological communities. Historical and geographical perspectives. Chicago, University of Chicago Press.

Dixon JR, SoINI P (1986) The reptiles of the upper Amazon Basin, Iquitos region, Peru. Milwaukee, Milwaukee Public Museum, 154p.

DiXon JR, WIEST-JR JA, CEI JM (1993) Revision of the Neotropical Snake Genus Chironius Fitzinger (Serpentes, Colubridae). Torino, Museo Regionale di Scienze Naturali, Monografie XIII, 279p.

Duellman WE (1978) The biology of an equatorial herpetofauna in Amazonian Ecuador. University of Kansas Museum Natural History, Miscellaneous Publication 65: 1-352.

Fernandes DS, Germano VJ, Fernandes R, Franco FL (2002) Taxonomic status of the lowland species of the Liophis cobella group with comments on the species of the Venenzuelan Tepuis (Serpentes, Colubridae). Boletim do Museu Nacional 481: 1-14.

França FGR, Mesquita DO, Colli R (2006) A checklist of snakes from Amazonian savannas in Brazil, housed in the Coleção Herpetológica da Universidade de Brasília, with new distribution records. Occasional Papers of The Sam Noble Oklahoma Museum of Natural History 17: 1-13.

França FGR, Mesquita DO, Nogueira CC, Araújo AFB (2008) Phylogeny and ecology determine morphological structure in a snake assemblage in the Central Brazilian Cerrado. Copeia 1: 23-38. doi: 10.1643/CH-05-034

Frota JG, Santos-Jr AP, Chalkidis HM, Guedes AG (2005) As serpentes da região do baixo rio Amazonas, oeste do Estado do Pará, Brasil (Squamata). Biociências 13(2): 211-220.

Goeldi E (1897) Die Eiervon 13 brasilianischen Reptilien, nebst Bemerkungen über Lebensund Fortpflanzungsweise letzteres. Zoologischer Jahresbericht 10: 640-76.

Goulding M, BARTHEM R, Ferreira E (2003) The Smithsonian atlas of the Amazon. Washington, D.C., Smithsonian Institution, 253p.

Grazziotin FG, Zaher H, Murphyc RW, Scrocchi G, Benavides MA, Zhang Y, Bonatto SL (2012) Molecular phylogeny of the New World Dipsadidae (Serpentes: Colubroidea): a reappraisal. Cladistics 1(2012): 1-23. doi: 10.1111/j.10960031.2012.00393.x

Junk WJ (1997) The central amazon Floodplain: Ecology of a pulsing system. Berlin, Springer, 529p. doi: 10.1007/9783-662-03416-3

Kovach WL (2009) Oriana - Circular Statistics for Windows. Wales, Kovach Computing Services, Pentraeth, version 3.

Krebs CJ (1989) Ecological Methodology. New York, Harper Collins Publishers, 654p.

Lima AMM, Oliveira LL, Fontinhas RL, Lima RJS (2005) Ilha de Marajó: Revisão Histórica Hidroclimatológica, Bacias Hidrográficas e Propostas de Gestão. Holos Environment 5(1): 65-80.

López MS, Giraldo AR (2003) Diet of the large water snake $H y$ drodynastes gigas (Colubridae) from northeast Argentina. Amphibia-Reptilia 25: 178-184. 
Luz-Agostinho KD, Agostinho AA, Gomes LC, Júlio-Jr HF, Fugi R (2009) Effects of footing regime on the feeding activity and body condition of piscivorous fish in the Upper paraná River floodplain. Brazilian Journal of Biology 69(2): 481-490.

MacArthur RW, Wilson EO (1967) The theory of island biogeography. Princeton, Princeton University Press, 206p.

MANLY BFJ (1994) Multivariate Statistical Methods: a primer. London, Chapman and Hall, 215p.

Marques OAV, ETerovick A, Endo W (2000) Seasonal activity of snakes in the Atlantic Forest in southeastern Brazil. Amphibia-Reptilia 22: 103-111.

Martins M, Oliveira ME (1999). Natural history of snakes in forests of the Manaus region, Central Amazonia, Brazil. Herpetological Natural History 6(2): 78-150.

Maschio GF, Prudente AlC, Lima AC, Feitosa DT (2007) Reproductive biology of Anilius scytale (Linnaeu, 1758) (Serpentes, Aniliidae) from eastern Amazonia, Brazil. South American Journal of Herpetology 2(3): 179-183.

Maschio GF, Santos-Costa MC, Prudente ALC (2009) Comunidades de serpentes da Região de Caxiuanã com avaliação da Eficiência dos Métodos de Captura, p. 589-603. In: LisboA PLB (Ed.). Caxiuanã: Desafios Pará a Conservação de Uma Floresta Nacional na Amazônia. Belém, Museu Paraense Emilio Goeldi.

Nascimento OR, Ávila-Pires TCS, Santos INF, Lima ACM (1991) Répteis de Marajó e Mexiana, Pará, Brasil. I. Revisão bibliográfica e novos registros. Boletim do Museu Paraense Emílio Goeldi 7: 25-41.

Okada EK, Agostinh AA, Petrere M, Penczak T (2003) Factors affecting fish diversity and abundance in drying ponds and lagoons in the upper Paraná River Brazil. Ecohydrology \& Hydrobiology 3(1): 97-110.

Palmuti CF, Cassimiro J, Bertoluci J (2009) Food habits of snakes from the RPPN Feliciano Miguel Abdala, an Atlantic Forest fragment of southeastern Brazil. Biota Neotropica 9(1): 263-269.

PIANKA ER (1966) Latitudinal gradients in species diversity: A review of concepts. American Naturalist 100: 33-46.

Pinheiro lC, Abe PS, Bitar Yoc, Albarelli LPP, Santos-Costa MC (2015) Composition and ecológica patterns of snake assemblages in an Amazon-Cerrado Trasition Zone in Brazil. Iheringia, Série Zoologia 105(2): 147-156. doi: 10.1590/1678-476620151052147156

Prado CPA, Uetanabaro M, Haddad CFB (2005) Breeding a tivity patterns, reproductive modes, and habit use by anurans (Amphibia) in a seasonal environment in the Pantanal, Brazil. Amphibia-Reptilia 26: 211-221.

Prudente ALC, SAntos-Costa MC (2005) Checklist of Snakes from "Estação Científica Ferreira Penna", Eastern Amazonia, Pará State, Brasil. Boletim do Museu Paraense Emílio Goeldi 1(2): 153:180.

Prudente AlC, Maschio GF, Yamashina CE, Santos-Costa MC (2007) Morphology, Reproductive Biology and diet of Dendrophidion dendrophis (Schlegel, 1837) (Serpentes, Colubridae) in Bra- zilian Amazon. South American Journal of Herpetology 2(1): 53-58.

Prudente AlC, Maschio GF, Santos-Costa MC (2010) Serpentes da Bacia Petrolífera de Urucu, município de Coari, Amazonas, Brasil. Acta Amazonica 40(2): 381-386.

Prudente AlC, Menks AC, Silva FM, Maschio GF (2014) Diet and reproduction of the Western Indigo Snake Drymarchon corais (Serpentes: Colubridae) from the Brazilian Amazon. Herpetology Notes 7: 99-108.

RADAM BRASIL (1974). Levantamento de recursos naturais. Geologia, geomorfologia, solos, vegetação e uso potencial da terra. Rio de Janeiro, Departamento Nacional de Produção Mineral, vol. 5.

Rocha WA, Prudente ALC (2010) Snake assemblege of Parque Nacional de Sete Cidades, State of Piaui, Brazil. South American Journal of Herpetology 5: 132-142. doi: 10.2994/057.005.0207

Rodrigues FS, Prudente ALC (2011) The snake assemblage (Squamata: Serpentes) of a Cerrado-Caatinga transition area in Castelo do Piaui, state of Piaui, Brazil. Zoologia 28(4): 440448. doi: 10.1590/S1984-46702011000400005

Santos-Costa MC, Prudente AlC, Di-Bernardo M (2006) Reproductive Biology of Tantilla melanocephala (Linnaeus, 1758) (Serpentes, Colubridae) from Eastern Amazonia, Brazil. Journal of Herpetology 40(4): 556-559.

Santos-Costa MC, Maschio GF, Prudente ALC (2015) Natural history of snakes from Floresta Nacional de Caxiuanã, eastern Amazonia, Brazil. Herpetology Notes 8: 69-98.

SAwaya RJ, Marques OAV, Maritns M (2008) Composição e história natural das serpentes de Cerrado de Itirapina, São Paulo, sudeste do Brasil. Neotropica 8(3): 127-149.

SiLva NJ, Cites JW (1995) Patterns of diversity of neotropical squamate reptile species with emphasis on the Brazilian Amazon and the conservation potential of indigenous reserves. Conservation Biology 9: 873-901.

SNEATH PHA, SOKAL RR (1973) Numerical Taxonomy: the principles and practice of numerical classification. San Francisco, Freeman, 573p.

Souza KR, Prudente ALC, Maschio GF (2014) Reproduction and diet of Imantodes cenchoa (Linnaeus, 1758) (Dipsadidae: Dipsadinae) from the Brazilian Amazon. Zoologia 31(1): 8-19. doi: 10.1590/S1984-46702014000100002

StaRACE F (1998) Guide dês serpents et amphisbènes de Guyane. Paris, Ibis Rouge Editions, 449p.

STRÜssmann C (2000) Herpetofauna, p. 153-189. In: IBAMA (Ed.) Fauna Silvestre na região do Rio Manso - MT. Brasília, Ministério do Meio Ambiente, Centrais Elétricas do Norte do Brasil.

Strüssmann C, SAzima I (1993) The snake assemblage of the Pantanal at Poconé, western Brazil. Studies on Neotropical Fauna and Environment 28(3): 157-168.

VITT LJ (1987) Communities, p. 335-365. In: Seigel RA, Collins JT, NovAK SS (Eds.). Snakes: Ecology and Evolutionary Biology. 
New York, McGraw-Hill Publishing.

VitT LJ, VANGILDER LD (1983) Ecology of a snakes community in northeastern Brazil. Amphibia-Reptilia 4: 273-296.

Yuki RN, SANTOS RM (1996) Snakes from Marajó and Mexiana islands, Pará state, Brazil. Boletim do Museu Paraense Emílio Goeldi 12: 41-53.

ZAR JH (2010) Bioestatistical Analysis. New Jersey, Prentice-Hall International, $5^{\text {th }}$ ed.

Appendix 1. Specimens examined, deposited in the Herpetological Collection of the MPEG and MZUSP. *Specimens were not in the MPEG collection.

Atractus natans: MPEG 18340, MPEG 18342, MPEG 18420, MPEG 18421

Atractus schach: MPEG 24960

Boa constrictor: MPEG 15187, MPEG 18018, MPEG 19634, MPEG 19635, MZUSP 3652, MZUSP 3653

Bothrops atrox: MPEG 17337, MPEG 17338, MPEG 17341, MPEG 17342, MPEG 17344, MPEG 17347, MPEG 17350, MPEG 17352, MPEG 17353, MPEG 17354, MPEG 17355, MPEG 17356, MPEG 17357, MPEG 17369, MPEG 18282, MPEG 18283, MPEG 18319, MPEG

18323, MPEG 18414, MPEG 18425, MPEG 18493, MPEG 18644, MPEG 18797, MPEG 18798, MPEG 18799, MPEG 19262, MPEG 19645, MPEG 20814, MPEG 20817, MPEG 21175, MPEG 21178, MPEG 23528, MPEG 24408, MPEG 24410, MPEG 24954, MPEG 25323 Chironius carinatus: MPEG 8666, MPEG 17716, MZUSP 3600, MZUSP 3601

Chironius exoletus: MPEG 16381, MPEG 18281

Chironius flavolineatus: MPEG 18341

Chironius fuscus: MPEG 319, MPEG 17349, MPEG 17363, MPEG 18280, MPEG 19327

Chironius multiventris: MPEG 18318

Clelia clelia: MPEG 18045

Corallus hortulanus: MPEG 18636, MPEG 18801, MPEG 19336*, MPEG 20812

Crotalus durissus: MPEG 17780, MPEG 18415, MPEG 18810, MPEG 20820, MPEG 24041

Dendrophidion dendrophis: MPEG 17364

Drepanoides anomalus: MPEG 18315

Drymarchon corais: MPEG 18185

Epicrates cenchria: MPEG 17345

Epicrates maurus: MPEG18043, MPEG 18044, MPEG 18130, MPEG 18131, MPEG 18164, MPEG 18175, MPEG 18192, MPEG 18193, MPEG 18194, MPEG 18254, MPEG 18255, MPEG 18256, MPEG 18261*, MPEG 18262, MPEG 18263, MPEG 18264, MPEG 18362, MPEG 18601, MPEG 19625, MPEG 19648, MZUSP 3599

Erythrolamprus almadensis: MPEG 18054, MPEG 18057, MPEG 18059, MPEG 18060, MPEG 18061, MPEG 18065, MPEG 18066, MPEG 18078, MPEG 18080, MPEG 18081, MPEG 18082, MPEG 18083, MPEG 18084, MPEG 18085, MPEG 18086, MPEG 18089, MPEG 18096, MPEG 18108, MPEG 18109, MPEG 18124

Erythrolamprus poecilogyrus: MPEG 643, MPEG 766, MPEG 767, MPEG 3266, MPEG 16534, MPEG 16535, MPEG 16536, MPEG 16825, MPEG 16826, MPEG 16934, MPEG 18010, MPEG 18030, MPEG 18031, MPEG 18032, MPEG 18033, MPEG 18034, MPEG 18035, MPEG 18036, MPEG 18037, MPEG 18038, MPEG 18039, MPEG 18040, MPEG 18041, MPEG 18042, MPEG 18047, MPEG 18048, MPEG 18049, MPEG 18050, MPEG 18051, MPEG 18052, MPEG 18053, MPEG 18055, MPEG 18056, MPEG 18062, MPEG 18063, MPEG 18064, MPEG 18067, MPEG 18069, MPEG 18075, MPEG 18076, MPEG 18079, MPEG 18087, MPEG 18088, MPEG 18090, MPEG 18091, MPEG 18092, MPEG 18093, MPEG 18094, MPEG 18095, MPEG 18097, MPEG 18098, MPEG 18099, MPEG 18101, MPEG 18102, MPEG 18106, MPEG 18110, MPEG 18111, MPEG 18112, MPEG 18113, MPEG 18114, MPEG 18116, MPEG 18117*, MPEG 18118, MPEG 18119, MPEG 18120, MPEG 18121, MPEG 18122, MPEG 18123, MPEG 18125, MPEG 18149, MPEG 18150, MPEG 18151, MPEG 18166, MPEG 18167, MPEG 18168, MPEG 18169, MPEG 18170, MPEG 18171, MPEG 18172, MPEG 18173, MPEG 18174, MPEG 18312, MPEG 18314, MPEG 18316, MPEG 18322, MPEG 18324, MPEG 18640, MPEG 18641, MPEG 18642, MPEG 18643, MPEG 19644, MPEG 21204, MPEG 24064, MZUSP 3617

Erythrolamprus reginae: MPEG 3263, MPEG 17366, MPEG 18165, MPEG 18321, MPEG 24959, MPEG 17365, MPEG 18287, MPEG 19331 Erythrolamprus taeniogaster: MPEG 322, MPEG 769, MPEG 3264, MPEG 17346, MPEG 18077, MPEG 18100, MPEG 18103, MPEG 18104, MPEG 18105, MPEG 18107, MPEG 18115, MPEG 18132, MPEG 18148, MPEG 18152, MPEG 18153, MPEG 18154, MPEG 18288, MPEG 18361, MPEG 19328, MPEG 19622, MPEG 19623, MPEG 19624, MPEG 19643, MPEG 21173, MPEG 21174, MPEG 21176, MPEG 21748, MPEG 21749, MPEG 21750, MPEG 21751, MPEG 24955, MPEG 24956, MZUSP 3603, MZUSP 3604

Erythrolamprus typhlus: MPEG 18422, MPEG 19329

Eunectes deschauenseei: MPEG 18019, MPEG 18029, MPEG 18068, MPEG 18126, MPEG 18127, MPEG 18128, MPEG 18129, MPEG 19632, MPEG 19633, MPEG 19638, MPEG 19639, MPEG 19640, MPEG 19641

Eunectes murinus: MPEG 19411*, MPEG 19412*, MPEG 19637, MZUSP 3654, MZUSP 3655, MZUSP 3656 
Helicops angulatus: MPEG 18416, MPEG 18417, MPEG 18418, MPEG 18419, MPEG 18423, MPEG 18424, MPEG 19337, MPEG 19617, MPEG 21197, MPEG 21198, MPEG 21199, MPEG 21205

Helicops hagmanni: MPEG 25325

Helicops leopardinus: MPEG 284, MPEG 285, MPEG 636, MPEG 637, MPEG 638, MPEG 639, MPEG 640, MPEG 641, MPEG 642, MPEG 17717, MPEG 17718, MPEG 17719, MPEG 17721, MPEG 18014, MPEG 18325, MPEG 18488, MPEG 18489, MPEG 18490, MPEG 18491, MPEG 18683, MPEG 19618, MPEG 19619, MPEG 19620, MPEG 19621, MZUSP 3605

Helicops polylepis: MPEG 321, MPEG 17720, MPEG 21177, MPEG 21179

Helicops trivittatus: MPEG 644*, MPEG 645, MPEG 8665, MPEG 18013, MPEG 19629, MPEG 19630, MPEG 19631, MPEG 20813

Hydrodynastes gigas: MPEG 18012, MPEG 18046, MPEG 18070, MPEG 18071, MPEG 18184, MPEG 19636, MPEG 21487

Hydrops martii: MPEG 25324

Imantodes cenchoa: MPEG 320, MPEG 16461, MPEG 18317, MPEG 20674*

Lachesis muta: MPEG 19335, MPEG 19338

Leptodeira annulata: MPEG 17724, MPEG 17785, MPEG 18338, MPEG 18339, MPEG 19263, MPEG 21181, MPEG 25322, MZUSP 3607

Leptophis ahaetulla: MPEG 17348, MPEG 17351, MPEG 17714*, MPEG 17722, MPEG 18016, MPEG 18326, MPEG 18637, MPEG 18638, MPEG 19330, MPEG 19332, MPEG 19333, MPEG 19334, MPEG 19626, MPEG 19627, MPEG 19628, MPEG 23529 , MZUSP 3619

Lygophis lineatus: MPEG 286, MPEG 287, MPEG 288, MPEG 768, MPEG 3261*, MPEG 3262, MPEG 16935, MPEG 17723, MPEG 18058, MPEG 18072*, MPEG 18073, MPEG 18074, MPEG 18684, MPEG 19616, MPEG 21180, MPEG 24411

Lygophis meridionalis: MPEG 20815, MPEG 20816

Mastigodryas bifossatus: MPEG 770*, MPEG 16531*, MPEG 16936*

Mastigodryas boddaerti: MPEG 17367, MPEG 18343, MPEG 18345

Micrurus filiformis: MPEG 17340, MPEG 18136, MPEG 24958, MZUSP 5095

Micrurus hemprichii: MPEG 20042, MPEG 24957

Micrurus surinamensis: MPEG 17336

Oxybelis aeneus: MPEG 17335, MZUSP 3608

Oxybelis fulgidus: MPEG 17339, MPEG 17368, MPEG 18285, MPEG 18344, MPEG 21206

Oxyrhopus melanogenys: MPEG 3265

Philodryas argentea: MPEG 17343, MPEG 17359, MPEG 17360, MPEG 17361

Philodryas viridissima: MPEG 17358*

Pseudoboa coronata: MPEG 18284*, MPEG 18313*, MPEG 24409, MPEG 24961

Psomophis joberti: MPEG 18320, MPEG 20795, MPEG 20796, MPEG 20818, MPEG 20819, MPEG 21203

Siphlophis cervinus: MPEG 19841

Siphlophis compressus: MPEG 18286

Taeniophallus quadriocellatus: MPEG 17362, MPEG 20487

Thamnodynastes lanei: MPEG 16532, MPEG 17715, MPEG 18311

Thamnodynaste sp: MPEG 16422*, MPEG 16533*, MPEG 18310*, MPEG 18327*, MPEG 18639*, MPEG 19615*, MPEG 20808, MPEG 20809, MPEG 20810, MPEG 20811, MPEG 21200, MPEG 21201, MPEG 21202

Amerotyphlops reticulatus: MPEG 18011, MPEG 18015, MPEG 23581, MPEG 23582

Submitted: 4 March 2015

Received in revised form: 30 September 2015

Accepted: 19 December 2015

Editorial responsibility: Darren Norris

Author contributions: Conceived and designed the experiments:

GMR GFM ALCP. Conducted the experiments: GMR. Analyzed

the data: GMR GFM ALCP. Wrote the paper: GMR GFM ALCP.

Competing interests: The authors have declared that no

competing interests exist. 